\title{
Evolution of transcription factor function as a mechanism for changing metazoan developmental gene regulatory networks
}

\author{
Alys M Cheatle Jarvela and Veronica F Hinman ${ }^{*}$
}

\begin{abstract}
The form that an animal takes during development is directed by gene regulatory networks (GRNs). Developmental GRNs interpret maternally deposited molecules and externally supplied signals to direct cell-fate decisions, which ultimately leads to the arrangements of organs and tissues in the organism. Genetically encoded modifications to these networks have generated the wide range of metazoan diversity that exists today. Most studies of GRN evolution focus on changes to cis-regulatory DNA, and it was historically theorized that changes to the transcription factors that bind to these cis-regulatory modules (CRMs) contribute to this process only rarely. A growing body of evidence suggests that changes to the coding regions of transcription factors play a much larger role in the evolution of developmental gene regulatory networks than originally imagined. Just as cis-regulatory changes make use of modular binding site composition and tissue-specific modules to avoid pleiotropy, transcription factor coding regions also predominantly evolve in ways that limit the context of functional effects. Here, we review the recent works that have led to this unexpected change in the field of Evolution and Development (Evo-Devo) and consider the implications these studies have had on our understanding of the evolution of developmental processes.
\end{abstract}

Keywords: Transcription factor, Gene regulatory network, Development, Novelty

\section{Introduction}

Gene regulatory networks (GRNs) explain the gene expression states that direct a cell to establish a particular fate [1]. In development, these models describe the mechanisms that take an egg and its localized maternal determinants to an organism with properly placed tissues and fully differentiated cells. GRNs are predominantly composed of intercellular signaling molecules, transcription factor proteins, and cis-regulatory module (CRM) DNA, but here we will focus on the transcription factor component. The interaction between a transcription factor and a specific binding site within a CRM allow for positive or negative influence on expression of a target gene. Because these networks instruct the specification of a particular cell type or structure, changes to these networks result in the evolution of animal morphology.

There has been much debate surrounding the mechanisms by which GRNs evolve. Changes to cis regulatory modules

\footnotetext{
*Correspondence: veronica@cmu.edu

Department of Biological Sciences, Carnegie Mellon University, 4400 5th Ave, Pittsburgh, PA 15213, USA
}

\section{Biomed Central

have historically been considered the dominant source of GRN evolution, and this idea continues to be supported by new data in the genomics era (reviewed in [2-4]). While it is difficult to identify and dissect CRMs and subsequently associate them with a discernible functional divergence, nevertheless, numerous examples have been unearthed (for example, [5-8]). In recent years, genome-wide experiments, such as ChIP-Seq [9] and computational approaches have been instrumental in understanding the contribution of regulatory DNA evolution. For example, using such methods, Schmidt and colleagues detected many instances of lineage-specific gains and losses of binding events, suggesting rapid turnover in cis regulatory sequences [10]. Additionally, conserved noncoding sequences, which frequently have regulatory functions, turn over quickly [11].

On the other hand, several lines of evidence suggest that transcription factors are incredibly well conserved over evolutionary time. The first indication of this comes from the now famous examples from the Hox transcription factor cluster. These transcription factors are conserved in both sequence and function, patterning the body axis of 
organisms as disparate as insects and vertebrates [12,13]. More recently, this has been shown to extend to cnidarians $[14,15]$. These initial discoveries were followed by numerous and particularly compelling functional-equivalence studies in which transcription factors from widely disparate taxa were shown to rescue knock-out phenotypes (for example, [16-18]). In fact, the realization that largely overlapping sets of transcription factors drive the development of essentially all metazoans surveyed lead to the concept of the 'toolkit for development' and the birth of Evo-Devo as a discipline [19,20].

Even prior to this breadth of experimental evidence in support of CRM change as the primary driver of GRN evolution, some theorized that this would be the case [21]. The logic of this argument is as follows: transcription factors are pleiotropic, meaning that they are multifunctional, and thus, mutations that might result in adaptive changes in one context will almost certainly be detrimental to the organism in others. Meanwhile, CRMs are highly modular. A single gene frequently will be regulated by a separate CRM in each of its temporal and spatial expression domains, and therefore one context can easily be altered without affecting the others. Even individual CRMs are modular. CRMs typically contain multiple binding sites for several different transcription factors, each of which can be modified individually. Therefore, it is commonly accepted that transcription factors are under much more constraint than CRMs and, as a result, are less free to evolve changes in sequence and function $[4,22]$.

More recently, it has been argued that transcription factors also have the capacity to be modular, and consequently could contribute to developmental GRN evolution more significantly than originally considered $[23,24]$. These authors maintained that many aspects of protein expression and structure permit protein evolution by reducing pleiotropy. For example, use of tissue-specific splice forms and changes to protein-protein interactions, which will only be relevant in tissues where both interacting proteins are expressed, both offer mechanisms to reduce the pleiotropy associated with transcription factor changes. Recent work has provided even more support for these ideas in addition to revealing unpredicted sources of modularity. Just as genomic approaches have allowed for increased understanding of the contributions of CRM mutations to GRN evolution, bioinformatic, genome-wide, and other novel techniques have also been instrumental to gaining a better insight into the ways in which transcription factors evolve. Here, we survey and synthesize recent experimental findings that support an underappreciated role for transcription factor change in GRN evolution. In particular, we focus on modular protein changes that seem to be favored by evolution, as previously demonstrated by the CRM paradigm, and therefore could occur in other systems. While modular changes will reduce the pleiotropy associated with transcription factor evolution, these changes may still impact the surrounding GRNs in different ways than do CRM changes. Therefore, greater understanding of how both of these GRN components evolve is necessary to understand how species diverge and novel structures are devised.

\section{Review}

\section{The structure and function of transcription factors are inherently modular}

The basic biochemical function of a transcription factor is twofold: (i) to recognize and bind a short, specific piece of DNA within a regulatory region, and (ii) to recruit or bind other proteins relevant to transcriptional regulation, such as other transcription factors, chromatin remodeling proteins, and general RNA polymerase machinery. The first function, DNA binding, directs the transcription factor to its target loci. The second allows the factor to elicit changes in transcriptional levels by influencing the stability of the transcriptional apparatus or the chromatin state. Combined, these functions enable transcription factors to influence gene expression. At the structural level, transcription factor proteins contain discrete domains for exerting these functions, known as DNA-binding domains and protein-protein interaction domains. Some have more than one of each, and others may perform both functions via a single domain. Because transcription factors have such functional units, which may individually acquire mutations and be lost or gained over time, they are modular just as CRMs are and, thus, have opportunities to evolve in ways that minimize pleiotropy.

In general, DNA binding domains are extremely well-conserved, but the rest of the protein readily diverges between homologs. For example, the aforementioned Hox genes were discovered on the basis of highly conserved homeobox DNA-binding domains [12]. Yet, McGinnis and colleagues noted that while there was $75 \%$ or better identity within this domain, their Hox paralogs of interest were essentially unalignable outside of this region. This finding has largely held true for other transcription factor families, for instance, basic helix-loop-helix (bHLH) [25], forkhead box (Fox) [26], and Ets [27] families. As we discuss later, this is not to say that DNA binding domains and properties do not evolve. However, it demonstrates that distinct regions of a transcription factor structure and function can be maintained even while the rest of the protein may be changing in ways that might independently impact function.

Here, we will first discuss the mechanisms that can lead to an increase in transcription factor functional diversity and then consider ways that these functions can also evolve context dependence. Both sets of mechanisms allow transcription factor change to be modular, and importantly, multiple mechanisms can be combined to 
reduce evolutionary constraint even more. This is depicted in Figure 1, where gene duplication, exon shuffling, and modular DNA binding all offer ways by which a transcription factor can take on new abilities (Figure 1A). Alternative splicing, protein-protein interactions, and post-translational modifications allow transcription factor coding changes to be limited to a particular spatiotemporal context (Figure 1B), especially since these are strongly coupled to the expression specificity provided by CRMs (Figure 1C). Each of these mechanisms will be discussed in this review, with a focus on new experimental findings, especially those that highlight transcription factor modularity.

\section{The rise and expansion of metazoan transcription factor families}

Many transcription factor families arose at the base of the metazoan lineage (reviewed in [28]) and many even predate metazoans [29]. However, these families have each undergone series of duplications and divergence, resulting in numerous homologs, which are an important source of novel material for building GRNs [30-32].

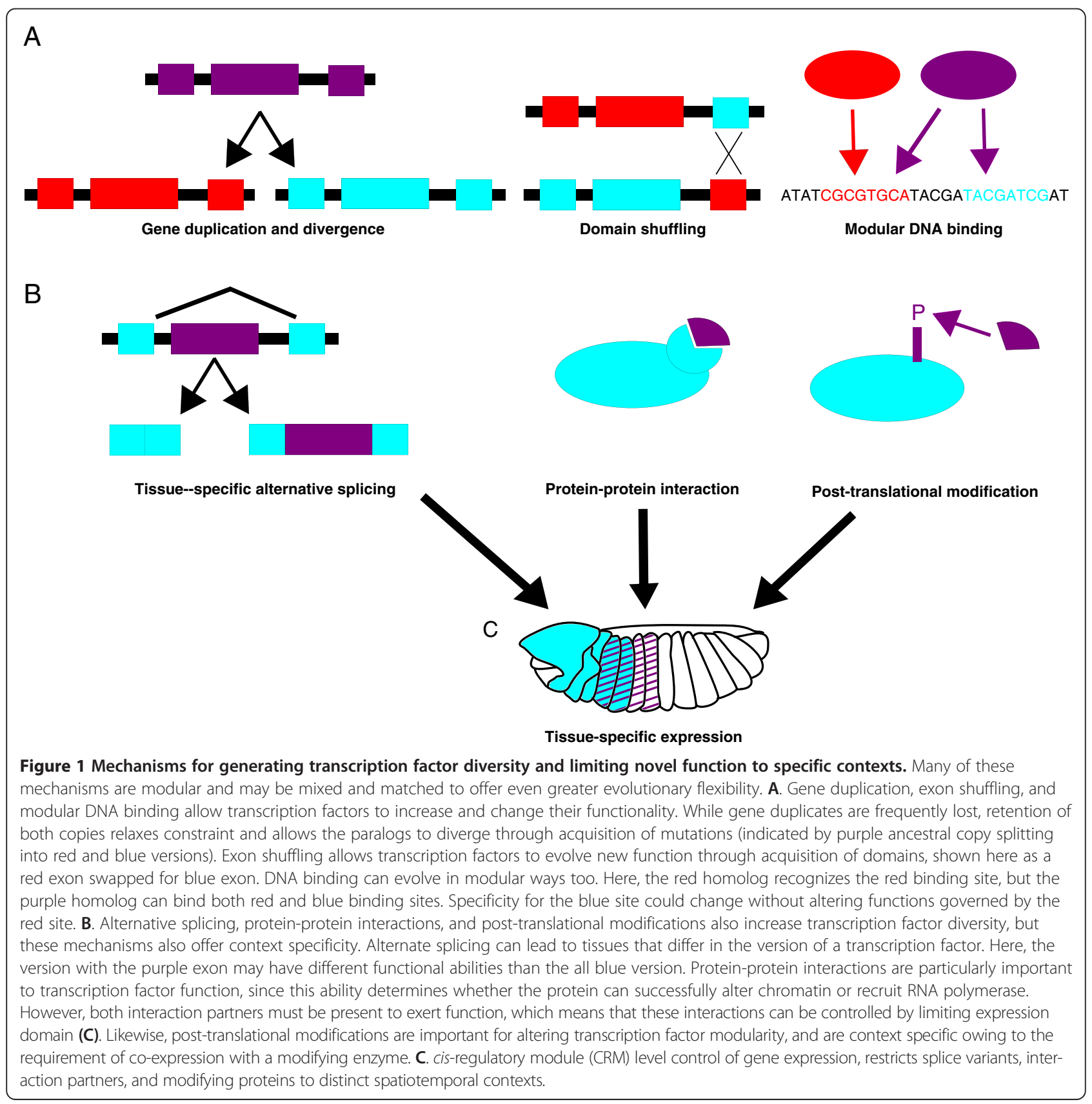


Additionally, an increase in the number and types of transcription factors available may have promoted the evolution of multicellularity; it has been suggested that even more transcription factors were added to the repertoire before the process of embryonic development could evolve [33]. Several important developmental transcription factors are not present in the sponge genome, suggesting that the creation of new transcription factors was critical to the evolution of bilaterians [34].

New transcription factor homologs are created in two ways. When species diverge from a common ancestor, each initially is endowed with the same collection of transcription factors, which generates orthologs. Following the split, each set will acquire mutations. Until a novel regulatory mechanism is devised, the orthologous proteins must execute the same tasks in each species as they did in the common ancestor. This means that orthologous transcription factors are under a great amount of constraint and are therefore thought to remain thoroughly conserved. Conversely, paralogs, transcription factors generated by gene duplication events, are much more free to evolve (Figure 1A) (reviewed in [24]). The new transcription factor, as a duplicate, can have several possible fates. Some duplicates are simply lost. Others take on some of the roles of the original transcription factor, lessening the burden on each copy and giving each copy more flexibility to change. This partitioning of function is known as subfunctionalization [35]. Finally, if one copy maintains all of the ancestral roles of the transcription factor, the other paralog will have essentially no constraint and can neofunctionalize. For example, vertebrate $\mathrm{A}-\mathrm{Myb}$ and $\mathrm{C}-\mathrm{Myb}$ are thought to have neofunctionalized after diverging from B-Myb; as a result B-Myb can rescue the single Drosophila Myb in functional-equivalence assays, but $\mathrm{A}$ and $\mathrm{C}-\mathrm{Myb}$ cannot [36]. This change occurred because the ancestor of $\mathrm{A}$ and $\mathrm{C}-\mathrm{Myb}$ acquired a new transcriptional activation domain. Additionally, A and C-Myb diverged from each other through subfunctionalization after they were generated by a gene duplication event, which allowed both to be preserved. C-Myb is SUMOylated at two lysines near its C-terminus, which stabilizes the protein and modifies its function in vivo [37]. These residues, and therefore this modification, are not conserved in A-Myb [38]. In this way, generation of paralogs results in modularity within a transcription factor family, because each paralog endows the others with greater freedom to change.

An interesting example of duplication and divergence occurs in the vertebrate steroid hormone receptors, a type of nuclear receptor transcription factor. These transcription factors split into two families with different abilities to recognize both hormone ligands and DNA sequences [39]. The extant vertebrate estrogen receptors appear to have maintained the ancestral capacity for both types of recognition, while the other clade of steroid receptors have evolved novel ligand and DNA specificity [40]. Because these new specificities evolved after gene duplication, the ancestral hormone signaling pathway was maintained.

A more extreme example of this is the nematode supplementary nuclear receptor family (supnrs) (reviewed [41]). In C. elegans there are 269 supnrs, thought to correspond most closely to vertebrate $\mathrm{Hnf} 4 \alpha$, although it is difficult to classify them due to highly diversified DNA binding and ligand binding domains [42,43]. As many supnrs are expressed, and therefore have not devolved into pseudogenes, it is thought that many have neofunctionalized or subfunctionalized. While some supnrs function very much like $\operatorname{Hnf} 4 \alpha$, others have evolved, potentially via changes to DNA and ligand binding domains, to function more like other metazoan nuclear receptors (reviewed in [41]).

Radiation of the supnrs is not an isolated example. Other transcription factor families also exhibit lineagespecific expansions, and so this is thought to be an important source of gene regulatory change (reviewed in [44]). Zinc finger transcription factor (ZNFs) subfamilies seem to be especially prone to this phenomenon. The zinc-finger associated domain (ZAD) subfamily underwent extensive lineage-specific expansion in the insect lineage, yet there is only one such protein in the vertebrate lineage [45]. Many of these insect-specific ZAD-ZNF transcription factors are associated with developmental processes and have been implicated in the evolution of the meroistic ovary.

Conversely, a different zinc-finger subfamily, the Krüppel-Associated-Box (KRAB-ZNF), radiated dramatically in tetrapod vertebrate lineages, while only one paralog, $\operatorname{Prdm} 9$, exists in invertebrates [46]. Many of these KRAB-ZNF proteins are expressed during early development and are crucial for executing epigenetic reprogramming and other early developmental tasks [46-48]. KRAB-ZNFs have been shown to be under positive selection and have acquired amino acid differences between humans and chimpanzees much faster than other genes [49]. Additionally, many KRAB-ZNFs are differentially expressed in the human brain compared to the chimpanzee brain, suggesting a role in the evolutionary divergence of brain development in these species [50]. Thus, expansions within the developmental toolkit are important to the evolution of developmental processes and potentially even the evolution of development as a process after multicellular animals emerged.

\section{Creating functional diversity among homologs}

Another mechanism for increasing modularity within transcription factor repertoires is exon shuffling. Exon shuffling allows for the creation of new genes by piecing 
together existing functional domains (Figure 1A). This mechanism has been known to create novel genetic toolkit components, and alter all aspects of the functionality of transcription factors. For example, while both the LIM and homeobox domains are ancient and can both be found in a variety of eukaryotes, the combination of two LIM domains and one homeobox to produce Lhx transcription factors is a metazoan innovation [51]. Secondary loss of this homeobox domain gave rise to the Lmo family of proteins, which affect gene expression by binding transcription factors since they cannot bind DNA on their own [51]. Therefore, the constituent parts of Lhx proteins can be gained and lost in a modular manner. Lhx genes have highly conserved roles in neurogenesis, and it has been suggested that they were co-opted into this process from an ancestral role in specifying primitive sensory cells [52]. Thus, the creation of this transcription factor family via domain shuffling was an important step in the evolution of neurogenic GRNs. Likewise, a comprehensive study of domain-shuffling in deuterostomes revealed that a handful of transcription factors in the vertebrate lineage acquired new transactivation domains that may have been important for the evolution of vertebrate-specific features [53]. Tandem duplication of exons can also accomplish this. For example, the DNA-binding abilities of KRAB-ZNFs are thought to be able to diverge by changing the number of zinc-finger domains in the protein [54]. Nowick and colleagues predict these changes will have effects on target genes known to be involved in neurogenesis, muscle, and limb development, all of which differ between humans and other primates. This mechanism also allowed the COE family of transcription factors to diverge through a tandem duplication of part of the helix-loop-helix domain at the base of the vertebrate lineage [55]. It is suggested that this change might allow vertebrate COE orthologs to make a wider variety of heterodimer pairings. Importantly, such rearrangements occur without necessarily altering the existing components, and therefore might take place without disrupting ancestral functions.

\section{Evolution of DNA-binding specificity}

Perhaps the most unexpected source of transcription factor adaptability is modular DNA-binding. This is surprising partly because functional-equivalence studies implied conserved DNA specificity of both orthologous $[17,18,56]$ and paralogous transcription factors $[57,58]$. Additionally, DNA-binding domains tend to be wellconserved at the sequence level. Instances of complete DNA-binding divergence have been uncovered, but they are quite rare [59-61]. The inability to assay transcription factor binding preferences in a sensitive and high-throughput way was for a long time a roadblock to such studies. PCR-based methods for discovering DNA-binding preference such as SELEX [62] recover only the highest affinity binding sites, and caused the misconception that protein-DNA recognition follows a simple one-to-one code. Only recently, it was realized that protein-DNA interactions are extraordinarily complex (reviewed in [63]). Newer technologies, such as proteinbinding microarrays $[64,65]$, are able to universally assess DNA-binding preference because all binding sites are assayed simultaneously without amplification steps as in SELEX. Because this technique uses purified proteins, it can be known with certainty that observed differences in sequence recognition between homologs are not due to modifications by, or interactions with, other proteins. This technique has therefore been crucial for recent works that have revealed modularity in transcription factor binding.

Initial studies that made use of protein-binding microarrays unearthed a few surprising findings. First, many transcription factors' binding preferences are best described by multiple position weight matrices rather than the one matrix [66-68]. These are commonly called primary and secondary motifs, where the primary motif is the most preferred. Collapsing these motifs into one position weight matrix obliterates important nucleotide interdependencies. For example, a transcription factor might bind well to motifs starting with AC or TG, but not AG or TC. However, a single position weight matrix depiction could imply that all of these combinations are equally preferred. Additionally, while closely related paralogs share highly similar primary binding sites, they frequently recognize different secondary binding sites (Figure 1A) [66-68]. Importantly, this phenomenon has been demonstrated in a variety of metazoan species (including mice, nematodes, and flies), and applies to many major transcription factor families (including Sox, Fox, ZNF, bHLH, Ets, and Homeodomain) [66-71]; therefore, these studies suggested an important and widespread source of transcription factor modularity that has only just been characterized in greater detail.

A recent study of yeast $\mathrm{C} 2 \mathrm{H} 2$ zinc finger paralogs also found modular differences in DNA-binding [72]. These proteins bind DNA using two adjacent zinc finger domains and can be divided into groups, in which a common canonical motif is bound by all members, and subgroups, which share an additional specific motif. Here, it was found that paralogs from the same group are able to adopt different conformations to recognize alternative binding sites; however the mechanism differs between subgroups. For example, one subgroup has evolved changes within both zinc finger domains that permit an alternate docking geometry, while another makes use of an $\mathrm{N}$-terminal region outside the zinc finger domains to stabilize alternative site binding. In all subgroups, both the canonical and alternative sites are bound with high affinity, indicating that recognition of the common canonical motif is not compromised by this 
plasticity. This is likely critical to maintenance of ancestral functions. Extensive cataloging of the Fox transcription factor family revealed flexibility in binding over evolutionary time too [73]. Some Fox proteins bind canonical primary and secondary motifs, some bind a completely different motif, termed FHL, and others are bispecific and therefore can use the primary, secondary, and FHL motifs. Intriguingly, preference for motifs like FHL and also for dual specificity has arisen multiple times within the Fox family, but never through changes to the DNA-binding helix. Instead, an N-terminal tail that allows for alternative structural configurations appears to be responsible for modular binding changes. These studies describe important new mechanisms that allow paralogous transcription factors to evolve while avoiding pleiotropic effects, in many cases by preserving binding to a canonical motif. This is a highly unexplored mechanism through which gene duplicates can acquire new function.

Orthologous transcription factors are under greater evolutionary constraint; therefore, until recently it was uncertain whether this type of modularity would extend to these genes. In addition to the differences in Fox paralog families described above, Nakagawa and colleagues also observed that different orthologs of yeast Fox3 exhibit substantial DNA binding diversity [73]. Some recognize the canonical primary and secondary motifs, others use the aforementioned FHL motif, and yet another subset recognizes a different variant, termed FVH. Fox3 orthologs that bind the FVH motif also have divergent amino acids in their DNA recognition helix. These observations suggested that orthologs may be able to make use of the same mechanisms as paralogs to diverge in DNA specificity. However these orthologs diverged between single-celled yeast species, and therefore may be under less constraint than the transcription factors used in metazoan development.

Recent work demonstrates that while metazoan developmental transcription factors may not diverge as dramatically as yeast orthologs, they do seem capable of exploiting modular divergence mechanisms used by paralogs [74]. In this study, it was found that orthologs of a t-box transcription factor, Tbr, from a sea urchin (Strongylocentrotus purpuratus) and a sea star (Patiria miniata) evolved differences in their secondary binding abilities. Interestingly this secondary motif is also different compared to what has been reported for the vertebrate ortholog of Tbr, Eomesodermin [66]. However, all three orthologs recognize the same primary motif despite 800 million years of divergence time [75]. The mechanism that allowed this change to evolve is not yet known, but these orthologs have differences in DNA-contacting amino acids, which might have caused changes in binding specificity. Interestingly, Tbr is known to have different developmental functions in the sea urchin compared to the sea star. In the sea star, Tbr has roles in the development of the endomesoderm and also in the ectoderm $[5,76,77]$. However, in the sea urchin, Tbr's only function is in skeletogenesis [78,79]. Changes to Tbr's DNA binding abilities over the course of echinoderm evolution may be responsible for differences in the developmental roles of this protein.

Several studies have demonstrated that these secondary and other non-canonical alternative binding sites are not only functional in vivo, but in many cases have distinct developmental tasks. Notably, in the case of Hedgehogresponsive genes used during Drosophila development, low-affinity, non-canonical alternative Cisites cannot be replaced by canonical, higher affinity sites as this results in a switch from activation to repression [80,81]. As a result, these sites convey important positional information across the anterior-posterior axis during development. In another example, it was found that differences in secondary motif specificity among homeodomain paralogs allows each to execute a particular regulatory program during Drosophila muscle development; all have the same primary motif and therefore would not be able to confer different myoblast identities without these unique secondary motifs [69]. Thus, secondary motifs are not an artifact of the protein-binding microarray technology, and exhibit in vivo functionality just as primary sites do. Because primary and secondary sites have distinct functions, the effects of changing binding to one type of site are less pleiotropic than changing binding to a solitary binding site. Importantly, since alternative binding sites can be gained and lost without affecting a conserved site [72-74], these developmental functions can be uncoupled and evolve independently, thus relieving constraint on developmental processes and allowing for more diverse cell types and structures to arise.

It has been suggested that use of high affinity primary and lower affinity secondary sites during development could be important to coordinate the timing of different developmental events through a temporal protein gradient [74]. For example, during eye development, proper timing of Pax6 expression is controlled by the affinity of the Prep1 binding sites within its enhancer [82]. The endogenous sites are low affinity, and replacing these with higher affinity sites causes Pax6 expression to begin too early. Heterochrony, or shifts in the rate or timing of developmental processes, is an important source of morphological differences between species (reviewed in $[83,84]$ ). Modular evolution of binding site preference and affinity could explain some cases where shifts in relative timing occur, because it allows for coupling and decoupling of processes coordinated by, but differentially responsive to, the same spatiotemporal protein gradient. 


\section{The contextual specificity of transcription factors is inherently modular}

In addition to having modular structure and function, transcription factors can also evolve reduced pleiotropy by limiting the spatiotemporal context of their functions. As mentioned previously, temporal control is crucial to the faithful execution of developmental programs, and shifts in timing can alter development and lead to the evolution of morphological changes. Thus limiting a transcription factor's action to a particular developmental period is important to the process. Likewise, control of the transcription factor's spatial domain is important for developmental processes. GRNs typically make use of combinatorial logic; thus, addition or subtraction of a constituent transcription factor results in alterations to where the GRN is active. Such changes can modify development and resulting morphology. Therefore, mechanisms are in place to ensure not only that transcription factors are expressed and active in particular developmental contexts, but also to allow the many processes they participate in to be uncoupled and evolve independently. The most well-known, and probably also the most common, mechanism for limiting and altering spatiotemporal contexts is by control of transcription factor expression through CRMs(reviewed in $[3,4]$ ). Here, we highlight ways in which modifications to transcription factor coding regions can allow for context specificity and, thus, also reduce pleiotropy.

\section{Context-dependent use of domains}

Alternative splicing can evolve to produce lineage-specific variants of transcription factors in a modular way from the existing structural composition. This is thought to be particularly useful in the evolution of developmental GRNs because different variants can be limited to a particular tissue or developmental stage (reviewed in [23]). This mechanism is reminiscent of CRM evolution, but offers an opportunity to change the functional ability of the protein through inclusion or exclusion of exons carrying protein-protein interaction or post-translational modification motifs, in addition to limiting the context of isoform expression. Alternative splicing has also been shown to be able to alter DNA-binding domain architecture and, potentially, also DNA-binding specificity in a tissue-specific manner [85]. More recently, Blekhman and colleagues used RNA-Seq to study transcript levels among three primate species and found that the expression of particular splice forms differs between lineages and sexes [86]. In Drosophila, sex-specific abdominal pigmentation patterns require gender-specific splice forms of the transcription factor Dsx, such that the female form activates gene expression and the male form represses expression from the same CRM [87]. Both splice forms use the first three exons of the Dsx gene, but the C-terminus of each form is sex-specific due to the retention of exon 4 in the female version, and 5 and 6 in the male version [88]. Interestingly, these splice forms differ in their ability to bind a transcriptional cofactor, Ix [89]. These examples demonstrate that the usage of transcription factor domains is modular and, thus, has the potential to be evolutionarily labile.

\section{Evolution of protein-protein interactions}

Transcription factors do not influence gene expression on their own, but do so as regulatory complexes mediated by interactions between the constituent transcription factors and cofactors. These interactions tend to be contextdependent; a particular protein-protein interaction will only be relevant when both interacting partners are present. The composition of a transcription factor complex is also guided by the types of binding sites present in the CRM, and so many transcription factors participate in multiple non-identical complexes and are able to form interactions with more than one other protein. Therefore, changes to such interactions are predicted to be minimally pleiotropic.

A well-known example critical to arthropod evolution is Ftz, which acquired novel cofactor interactions that changed the function of this transcription factor from homeotic to pair-rule segmentation factor [90]. This occurred through several steps. Change to Ftz's expression domain via CRM evolution was important, but so were changes to the protein coding region. These changes resulted in the loss of an ancestral interaction peptide motif, YPWM, which is required for interaction with Exdand homeotic function, and gain of a new LXXLL motif, which created an interaction with Ftz-F1. The latter confers most segmentation function, although the $\mathrm{N}$ terminal arm of the homeodomain also participates. More recently, it was shown that this is not a simple case of drastic changes in a particular lineage. Rather, the YPWM homeotic potential motif evolved into stronger and weaker variants of the ancestral sequence throughout the arthropod clade [91]. While YPWM does not hamper the functionality of the LXXLL motif, it does reduce the residual segmentation ability of Ftz variants that lack LXXLL and, therefore, may impact the evolution of particular Ftz lineages. This suggests an inherent flexibility in this YPWM binding motif that could be co-opted by GRNs to create novelty at other points in the evolutionary trajectory of these organisms. It also suggests that intermediate forms of an adaptive protein change need not be catastrophic to development, which is a common argument against transcription factor evolution as an important component of GRN evolution.

Newly evolved interaction motifs are also able to change the magnitude of an existing function. Throughout bilaterians, the transcription factor Engrailed (En) interacts with a co-repressor Groucho (Gro), usually through a 
well-conserved motif [92,93]. However certain groups of insects, namely dipterans and lepidopterans, have an additional, novel Gro interaction motif. This novel motif strengthens the interaction between Gro and En and, as a result, augments En's existing repressive abilities rather than conferring a novel function on En [94]. An advantage of changing GRNs through CRMs includes the ability to increase or decrease the quantity of a gene product and thus enhance or tone-down its function. This work suggests that the evolution of protein-protein interaction motifs is capable of producing quantitative changes as well.

Importantly, changes to protein-protein interactions can occur without major disruptions of the existing protein-protein interaction domain. Brayer and colleagues discovered that an important new interaction evolved between Hoxa11 and Foxola in placental mammals without actually changing the ancestral binding interface [95]. These genes are both crucial to the regulation of gene expression in endometrial stromal cells, and adaptive changes in Hoxa11 had already been shown to be a driving force in evolution of pregnancy in mammals [96]. Without Foxola, Hoxa11 represses the expression of pregnancy-related genes instead of activating them, so the advent of the Foxola/Hoxa11 interaction is key to the origin of this novelty [97]. Interestingly, the binding interface of these proteins did not change; in fact, Foxola had not evolved much at all as evidenced by the fact that eutherian Hoxa11 is able to interact with non-mammalian orthologs of Foxo1a [95]. This is critical because Hoxa11 interacts with Foxola via its homeodomain, which is used in other essential functions of this transcription factor such as DNA-binding. The authors suggest that the causative amino-acid changes most likely produced a conformational difference in the protein in the eutherian lineage that makes a pre-existing binding interface accessible to Foxola [95].

These case studies highlight the previously underappreciated versatility of transcription factor coding region changes, in addition to offering a mechanism for limiting the context of the evolved transcription factor's function. Furthermore, they reveal that mutations to transcription factors can accomplish some of the same advantageous functions of CRMs, such as the ability to tweak target gene transcriptional output. Finally, they demonstrate that there are many ways to alter a transcription factor without abolishing ancestral function, such as through changing protein conformation as opposed to the sequence of the functional domain.

\section{Evolution of post-translational modifications}

Post-translational modifications are a common way to increase protein functional diversity. They are of particular interest to those seeking to understand how transcription factors may evolve while avoiding pleiotropy because they are known to regulate the location, longevity, and activity of proteins. They can also allow for alternate protein structure and enhance or prevent protein-protein interactions and DNA-binding (reviewed in $[98,99]$ ). Thus, as is the case for CRMs, the effects of mutations to post-translational modifications can easily be limited to a particular developmental context. Some types of modification, such as phosphorylation, are reversible, and therefore offer even more flexibility.

Moreover, new modification sites evolve rapidly. A comprehensive bioinformatics screen identified over two-hundred ubiquitylation sites that arose in the human lineage since it split from other primates [100]. A similar study also found 37 human-specific phosphorylation sites [101]. Interestingly, it has been suggested that a human-specific protein kinase $C$ phosphorylation site has evolved in the Foxp2 transcription factor, which is important for cortical development and has been implicated in the evolution of speech in humans [102]. It is thought that this modification allowed Foxp2 to enhance its neurogenic function, since the human version has a gain-of-function phenotype in transgenic mice [103].

It is unsurprising then that recent work has found compelling connections between novel post-translational modification sites within transcription factors and the evolution of new features. For example, Ubx, a Hox transcription factor, is expressed in the limb primordia of both insects and crustaceans. Thus, alteration of the Ubx protein explains differences in appendage number between different groups of arthropods rather than CRM level changes [104]. Taghli-Lamallem and colleagues found that an important difference in Ubx among arthropods involves loss of CK2 phosphorylation sites in the insect lineage [105]. Ubx represses the expression of $D l l$, which also results in repression of limb formation. They demonstrated that phosphorylation of CK2 sites in crustaceans interferes with the ability of Ubx to repress $D l l$, and as a result more appendages form in crustaceans compared to insects. The molecular consequence of phosphorylating these sites is unknown, but there are precedents for phosphorylation affecting DNA-binding of Hox proteins and also their protein-protein interactions [106,107].

Another interesting example entails evolution of pregnancy in mammals, due in part to changes in phosphorylation of Cebp $\beta$ [108]. This work demonstrated that a mere three amino-acid changes in an internal regulatory domain, resulting in the loss of two ancestral phosphorylation sites and the gain of a new one elsewhere, completely changed how this transcription factor responds to cAMP signaling. Phosphorylation of the novel site by Gsk-3 $\beta$ is required for $\operatorname{Cebp} \beta$ to activate the expression of prolactin, an important pregnancy hormone. Developmental 
GRNs integrate both signaling pathways and transcription factors, and so alteration of the post-translational modifications that connect them offers an attractive way of modifying developmental GRNs.

\section{Conclusions}

Transcription factor coding changes are becoming a theoretically more accepted source of GRN evolution, but there are still only a few studies documenting specific changes and tying those to developmental novelties. Many of the studies we have discussed in this review suggest interesting ways GRN evolution can occur via transcription factor change, but further study is still required in order to understand the full mechanism. As these experimental examples continue to increase, we will be able to decipher what impact these changes have on the wiring of their GRNs and how this might differ from CRM mutations. The original logic supporting CRM mutations over transcription factor changes would suggest that the former are ideally suited to alter the expression of a particular gene and potentially also its downstream targets within a tissue or cell-type, while changes to transcription factors will have broader effects, changing the regulation of large sets of target genes across the organism. The experimental evidence described here points to incremental and modular transcription factor mutations being favored by evolution, and latent motifs and abilities becoming more pronounced or reduced over time. Thus, in many ways, transcription factors evolve in ways that are very reminiscent of CRM evolution in that both use modularity to circumvent pleiotropy. However, it is important to realize that their effects on the surrounding GRNs are potentially not equal. Each type of change may be more ideal for driving different types of GRN changes and developing different types of novelty. More information about both types of change is required to tease out this discrepancy. On the other hand, several recent works suggest that CRM and transcription factor mutations may generally operate together $[91,109,110]$. Additional work will reveal whether such cooperative changes to GRNs are the exception, the rule, or simply another option in creating diverse GRNs, a myriad of developmental processes, and seemingly endless animal forms.

\section{Abbreviations \\ bHLH: basic helix-loop-helix; CRM: cis regulatory module; Evo-Devo: Evolution and Development; Fox: forkhead box; GRN: gene regulatory network; KRAB: Krüppel-Associated-Box; Supnrs: supplementary nuclear receptors; ZAD: zinc-finger associated domain; ZNF: zinc finger.}

\section{Competing interests}

The authors declare that they have no competing interests.

Authors' contributions

$\mathrm{ACJ}$ and $\mathrm{VH}$ wrote, read, and approved this manuscript.

\section{Acknowledgements}

The authors thank Dr. Greg Cary as well as four anonymous reviewers for their critical reading of this manuscript. This work was supported by funding from NSF IOS 0844948 and IOS 1024811 awarded to VFH.

Received: 5 November 2014 Accepted: 18 December 2014 Published: 29 January 2015

References

1. Davidson EH. The Regulatory Genome: Gene Regulatory Networks In Development And Evolution. Burlington, MA: Academic Press; 2010.

2. Rubinstein M, de Souza FSJ. Evolution of transcriptional enhancers and animal diversity. Philos Trans R Soc Lond B Biol Sci. 2013;368:20130017.

3. Wittkopp PJ, Kalay G. Cis-regulatory elements: molecular mechanisms and evolutionary processes underlying divergence. Nat Rev Genet. 2012;13:59-69.

4. Wray GA. The evolutionary significance of cis-regulatory mutations. Nat Rev Genet. 2007:8:206-16.

5. Hinman VF, Nguyen A, Davidson EH. Caught in the evolutionary act: precise cis-regulatory basis of difference in the organization of gene networks of sea stars and sea urchins. Dev Biol. 2007:312:584-95.

6. Arnoult L, Su KFY, Manoel D, Minervino C, Magriña J, Gompel N, et al. Emergence and Diversification of Fly Pigmentation Through Evolution of a Gene Regulatory Module. Science. 2013;339:1423-6.

7. Guerreiro I, Nunes A, Woltering JM, Casaca A, Nóvoa A, Vinagre T, et al. Role of a polymorphism in a Hox/Pax-responsive enhancer in the evolution of the vertebrate spine. Proc Natl Acad Sci. 2013:110:10682-6.

8. Rogers WA, Salomone JR, Tacy DJ, Camino EM, Davis KA, Rebeiz M, et al. Recurrent modification of a conserved cis-regulatory element underlies fruit fly pigmentation diversity. PLoS Genet. 2013;9:e1003740.

9. Johnson DS, Mortazavi A, Myers RM, Wold B. Genome-wide mapping of in vivo protein-DNA interactions. Science. 2007:316:1497-502.

10. Schmidt D, Wilson MD, Ballester B, Schwalie PC, Brown GD, Marshall A, et al. Five-vertebrate ChIP-seq reveals the evolutionary dynamics of transcription factor binding. Science. 2010;328:1036-40.

11. Meader S, Ponting CP, Lunter G. Massive turnover of functional sequence in human and other mammalian genomes. Genome Res. 2010;20:1335-43.

12. McGinnis W, Garber RL, Wirz J, Kuroiwa A, Gehring WJ. A homologous protein-coding sequence in Drosophila homeotic genes and its conservation in other metazoans. Cell. 1984;37:403-8.

13. Duboule $D$, Dollé $P$. The structural and functional organization of the murine HOX gene family resembles that of Drosophila homeotic genes. EMBO J. 1989:8:1497-505

14. Ryan JF, Baxevanis AD. Hox, Wnt, and the evolution of the primary body axis: insights from the early-divergent phyla. Biol Direct. 2007:2:37.

15. DuBuc TQ, Ryan JF, Shinzato C, Satoh N, Martindale MQ. Coral Comparative Genomics Reveal Expanded Hox Cluster in the Cnidarian-Bilaterian Ancestor. Integr Comp Biol. 2012;52:835-41.

16. Halder G, Callaerts P, Gehring WJ. Induction of ectopic eyes by targeted expression of the eyeless gene in Drosophila. Science. 1995;267:1788-92.

17. McGinnis N, Kuziora MA, McGinnis W. Human Hox-4.2 and Drosophila deformed encode similar regulatory specificities in Drosophila embryos and larvae. Cell. 1990;63:969-76.

18. Wang VY, Hassan BA, Bellen HJ, Zoghbi HY. Drosophila atonal fully rescues the phenotype of Math1 null mice: new functions evolve in new cellular contexts. Curr Biol CB. 2002:12:1611-6.

19. Carroll SB. Endless Forms Most Beautiful: The New Science of Evo Devo and the Making of the Animal Kingdom. New York, NY: Norton \& Company: W. W; 2005.

20. Carroll SB. Evo-devo and an expanding evolutionary synthesis: a genetic theory of morphological evolution. Cell. 2008;134:25-36.

21. Britten RJ, Davidson EH. Repetitive and non-repetitive DNA sequences and a speculation on the origins of evolutionary novelty. Q Rev Biol. 1971;46:111-38.

22. Stern DL. Evolutionary developmental biology and the problem of variation. Evol Int J Org Evol. 2000;54:1079-91.

23. Lynch VJ, Wagner GP. Resurrecting the role of transcription factor change in developmental evolution. Evol Int J Org Evol. 2008;62:2131-54.

24. Hoekstra HE, Coyne JA. The locus of evolution: evo devo and the genetics of adaptation. Evol Int J Org Evol. 2007;61:995-1016.

25. Castanon I, Baylies MK. A Twist in fate: evolutionary comparison of Twist structure and function. Gene. 2002;287:11-22. 
26. Kaestner KH, Knochel W, Martinez DE. Unified nomenclature for the winged helix/forkhead transcription factors. Genes Dev. 2000;14:142-6.

27. Laudet V, Hänni C, Stéhelin D, Duterque-Coquillaud M. Molecular phylogeny of the ETS gene family. Oncogene. 1999;18:1351-9.

28. Degnan BM, Vervoort M, Larroux C, Richards GS. Early evolution of metazoan transcription factors. Curr Opin Genet Dev. 2009;19:591-9.

29. Sebé-Pedrós A, de Mendoza A, Lang BF, Degnan BM, Ruiz-Trillo I. Unexpected repertoire of metazoan transcription factors in the unicellular holozoan Capsaspora owczarzaki. Mol Biol Evol. 2011;28:1241-54.

30. Holland PWH. Evolution of homeobox genes. Wiley Interdiscip Rev Dev Biol. 2013;2:31-45.

31. Pérez JC, Fordyce PM, Lohse MB, Hanson-Smith V, DeRisi JL, Johnson AD. How duplicated transcription regulators can diversify to govern the expression of nonoverlapping sets of genes. Genes Dev. 2014;28:1272-7.

32. Teichmann SA, Babu MM. Gene regulatory network growth by duplication. Nat Genet. 2004;36:492-6.

33. De Mendoza A, Sebé-Pedrós A, Šestak MS, Matejcic M, Torruella G, Domazet-Loso T, et al. Transcription factor evolution in eukaryotes and the assembly of the regulatory toolkit in multicellular lineages. Proc Natl Acad Sci U S A. 2013;110:E4858-66.

34. Srivastava M, Simakov O, Chapman J, Fahey B, Gauthier MEA, Mitros T, et al. The Amphimedon queenslandica genome and the evolution of animal complexity. Nature. 2010;466:720-6.

35. Force A, Lynch M, Pickett FB, Amores A, Yan YL, Postlethwait J. Preservation of duplicate genes by complementary, degenerative mutations. Genetics. 1999;151:1531-45.

36. Davidson CJ, Tirouvanziam R, Herzenberg LA, Lipsick JS. Functional evolution of the vertebrate Myb gene family B-Myb, but neither A-Myb nor c-Myb, complements Drosophila Myb in hemocytes. Genetics. 2005;169:215-29.

37. Davidson CJ, Guthrie EE, Lipsick JS. Duplication and maintenance of the Myb genes of vertebrate animals. Biol Open. 2013;2:101-10.

38. Ganter B, Lipsick JS. Myb and Oncogenesis. In: Vande Woude GF, Klein G, editors. Advances in Cancer Research, vol. 76. San Diego, CA: Academic Press; 1999. p. 21-60.

39. Mckeown AN, Bridgham JT, Anderson DW, Murphy MN, Ortlund EA, Thornton JW. Evolution of DNA specificity in a transcription factor family produced a new gene regulatory module. Cell. 2014:159:58-68.

40. Eick GN, Colucci JK, Harms MJ, Ortlund EA, Thornton JW. Evolution of minimal specificity and promiscuity in steroid hormone receptors. PLoS Genet. 2012;8:e1003072.

41. Kostrouchova M, Kostrouch Z. Nuclear receptors in nematode development: natural experiments made by a phylum. Biochim Biophys Acta BBA - Gene Regul Mech. 2014; doi: 10.1016/j.bbagrm.2014.06.016.

42. Robinson-Rechavi M, Maina CV, Gissendanner CR, Laudet V, Sluder A. Explosive lineage-specific expansion of the orphan nuclear receptor HNF4 in nematodes. J Mol Evol. 2005;60:577-86.

43. Sluder AE, Mathews SW, Hough D, Yin VP, Maina CV. The nuclear receptor superfamily has undergone extensive proliferation and diversification in nematodes. Genome Res. 1999;9:103-20.

44. Nowick K, Stubbs L. Lineage-specific transcription factors and the evolution of gene regulatory networks. Brief Funct Genomics. 2010;9:65-78.

45. Chung $H-R$, Löhr $U$, Jäckle $H$. Lineage-specific expansion of the zinc finger associated domain ZAD. Mol Biol Evol. 2007;24:1934-43.

46. Liu H, Chang L-H, Sun Y, Lu X, Stubbs L. Deep vertebrate roots for mammalian zinc finger transcription factor subfamilies. Genome Biol Evol. 2014;6:510-25.

47. Corsinotti A, Kapopoulou A, Gubelmann C, Imbeault M, Santoni de Sio FR, Rowe HM, et al. Global and stage specific patterns of Krüppel-associated-box zinc finger protein gene expression in murine early embryonic cells. PLoS One. 2013;8:e56721

48. Quenneville S, Turelli P, Bojkowska K, Raclot C, Offner S, Kapopoulou A, et al. The KRAB-ZFP/KAP1 system contributes to the early embryonic establishment of site-specific DNA methylation patterns maintained during development. Cell Rep. 2012;2:766-73.

49. Bustamante CD, Fledel-Alon A, Williamson S, Nielsen R, Todd Hubisz M, Glanowski S, et al. Natural selection on protein-coding genes in the human genome. Nature. 2005:437:1153-7.

50. Nowick K, Gernat T, Almaas E, Stubbs L. Differences in human and chimpanzee gene expression patterns define an evolving network of transcription factors in brain. Proc Natl Acad Sci. 2009;106:22358-63.
51. Koch BJ, Ryan JF, Baxevanis AD. The diversification of the LIM superclass at the base of the Metazoa increased subcellular complexity and promoted multicellular specialization. PLoS ONE. 2012;7:e33261.

52. Srivastava M, Larroux C, Lu DR, Mohanty K, Chapman J, Degnan BM, et al. Early evolution of the LIM homeobox gene family. BMC Biol. 2010;8:4.

53. Kawashima T, Kawashima S, Tanaka C, Murai M, Yoneda M, Putnam NH, et al. Domain shuffling and the evolution of vertebrates. Genome Res. 2009:19:1393-403

54. Nowick K, Fields C, Gernat T, Caetano-Anolles D, Kholina N, Stubbs L. Gain, loss and divergence in primate zinc-finger genes: a rich resource for evolution of gene regulatory differences between species. PLoS One. 2011;6:e21553.

55. Daburon V, Mella S, Plouhinec J-L, Mazan S, Crozatier M, Vincent A. The metazoan history of the COE transcription factors. Selection of a variant HLH motif by mandatory inclusion of a duplicated exon in vertebrates. BMC Evol Biol. 2008;8:131.

56. Pocock R, Mione M, Hussain S, Maxwell S, Pontecorvi M, Aslam S, et al. Neuronal function of Tbx20 conserved from nematodes to vertebrates. Dev Biol. 2008;317:671-85.

57. Gao Y, Lan Y, Ovitt CE, Jiang R. Functional equivalence of the zinc finger transcription factors Osr1 and Osr2 in mouse development. Dev Biol. 2009;328:200-9.

58. Hoser M, Potzner MR, Koch JMC, Bösl MR, Wegner M, Sock E. Sox12 deletion in the mouse reveals nonreciprocal redundancy with the related Sox4 and Sox11 transcription factors. Mol Cell Biol. 2008;28:4675-87.

59. Hanes SD, Brent R. DNA specificity of the bicoid activator protein is determined by homeodomain recognition helix residue 9 . Cell. 1989:57:1275-83.

60. Baker $C R$, Tuch BB, Johnson AD. Extensive DNA-binding specificity divergence of a conserved transcription regulator. Proc Natl Acad Sci U S A. 2011;108:7493-8.

61. Sayou C, Monniaux M, Nanao MH, Moyroud E, Brockington SF, Thévenon E, et al. A promiscuous intermediate underlies the evolution of LEAFY DNA binding specificity. Science. 2014;343:645-8.

62. Oliphant AR, Brandl CJ, Struhl K. Defining the sequence specificity of DNA-binding proteins by selecting binding sites from random-sequence oligonucleotides: analysis of yeast GCN4 protein. Mol Cell Biol. 1989;9:2944-9.

63. Siggers T, Gordân R. Protein-DNA binding: complexities and multi-protein codes. Nucleic Acids Res. 2014;42:2099-111.

64. Berger MF, Bulyk ML. Protein binding microarrays (PBMs) for rapid, high-throughput characterization of the sequence specificities of DNA binding proteins. Methods Mol Biol Clifton NJ. 2006;338:245-60.

65. Berger MF, Philippakis AA, Qureshi AM, He FS, Estep PW, Bulyk ML. Compact, universal DNA microarrays to comprehensively determine transcription-factor binding site specificities. Nat Biotechnol. 2006;24:1429-35.

66. Badis G, Berger MF, Philippakis AA, Talukder S, Gehrke AR, Jaeger SA, et al. Diversity and complexity in DNA recognition by transcription factors. Science. 2009;324:1720-3

67. Gordân R, Murphy KF, McCord RP, Zhu C, Vedenko A, Bulyk ML. Curated collection of yeast transcription factor DNA binding specificity data reveals novel structural and gene regulatory insights. Genome Biol. 2011;12:R125.

68. Zhu C, Byers KJRP, McCord RP, Shi Z, Berger MF, Newburger DE, et al. High-resolution DNA-binding specificity analysis of yeast transcription factors. Genome Res. 2009;19:556-66.

69. Busser BW, Shokri L, Jaeger SA, Gisselbrecht SS, Singhania A, Berger MF, et al. Molecular mechanism underlying the regulatory specificity of a Drosophila homeodomain protein that specifies myoblast identity. Dev Camb Engl. 2012;139:1164-74.

70. Wei G-H, Badis G, Berger MF, Kivioja T, Palin K, Enge M, et al. Genome-wide analysis of ETS-family DNA-binding in vitro and in vivo. EMBO $J$. 2010;29:2147-60.

71. Grove CA, De Masi F, Barrasa MI, Newburger DE, Alkema MJ, Bulyk ML, et al. A multiparameter network reveals extensive divergence between $C$. elegans bHLH transcription factors. Cell. 2009;138:314-27.

72. Siggers T, Reddy J, Barron B, Bulyk ML. Diversification of transcription factor paralogs via noncanonical modularity in $\mathrm{C} 2 \mathrm{H} 2$ zinc finger DNA binding. Mol Cell. 2014;55:640-8.

73. Nakagawa S, Gisselbrecht SS, Rogers JM, Hartl DL, Bulyk ML. DNA-binding specificity changes in the evolution of forkhead transcription factors. Proc Natl Acad Sci. 2013;110:12349-54. 
74. Cheatle Jarvela AM, Brubaker L, Vedenko A, Gupta A, Armitage BA, Bulyk ML, et al. Modular evolution of DNA binding preference of a Tbrain transcription factor provides a mechanism for modifying gene regulatory networks. Mol Biol Evol. 2014;31:2672-88.

75. Hedges SB, Dudley J, Kumar S. TimeTree: a public knowledge-base of divergence times among organisms. Bioinforma Oxf Engl. 2006;22:2971-2.

76. Hinman VF, Davidson EH. Evolutionary plasticity of developmental gene regulatory network architecture. Proc Natl Acad Sci U S A. 2007;104:19404-9.

77. McCauley BS, Weideman EP, Hinman VF. A conserved gene regulatory network subcircuit drives different developmental fates in the vegetal pole of highly divergent echinoderm embryos. Dev Biol. 2010;340:200-8.

78. Croce J, Lhomond G, Lozano JC, Gache C. ske-T, a T-box gene expressed in the skeletogenic mesenchyme lineage of the sea urchin embryo. Mech Dev. 2001;107:159-62

79. Oliveri P, Carrick DM, Davidson EH. A regulatory gene network that directs micromere specification in the sea urchin embryo. Dev Biol. 2002;246:209-28.

80. Parker DS, White MA, Ramos Al, Cohen BA, Barolo S. The cis-regulatory logic of Hedgehog gradient responses: key roles for gli binding affinity, competition, and cooperativity. Sci Signal. 2011;4:ra38.

81. Ramos Al, Barolo S. Low-affinity transcription factor binding sites shape morphogen responses and enhancer evolution. Philos Trans R Soc B Bio Sci. 2013;368:20130018.

82. Rowan S, Siggers T, Lachke SA, Yue Y, Bulyk ML, Maas RL. Precise temporal control of the eye regulatory gene Pax6 via enhancer-binding site affinity. Genes Dev. 2010;24:980-5.

83. Keyte AL, Smith KK. Heterochrony and developmental timing mechanisms: Changing ontogenies in evolution. Semin Cell Dev Biol. 2014;34C:99-107.

84. Smith KK. Time's arrow: heterochrony and the evolution of development. Int J Dev Biol. 2003:47:613-21.

85. Taneri B, Snyder B, Novoradovsky A, Gaasterland T. Alternative splicing of mouse transcription factors affects their DNA-binding domain architecture and is tissue specific. Genome Biol. 2004;5:R75.

86. Blekhman R, Marioni JC, Zumbo P, Stephens M, Gilad Y. Sex-specific and lineage-specific alternative splicing in primates. Genome Res. 2010;20:180-9.

87. Williams TM, Selegue JE, Werner T, Gompel N, Kopp A, Carroll SB. The regulation and evolution of a genetic switch controlling sexually dimorphic traits in drosophila. Cell. 2008;134:610-23.

88. Burtis KC, Baker BS. Drosophila doublesex gene controls somatic sexua differentiation by producing alternatively spliced mRNAs encoding related sex-specific polypeptides. Cell. 1989;56:997-1010.

89. Garrett-Engele CM, Siegal ML, Manoli DS, Williams BC, Li H, Baker BS intersex, a gene required for female sexual development in Drosophila, is expressed in both sexes and functions together with doublesex to regulate terminal differentiation. Dev Camb Engl. 2002;129:4661-75.

90. Löhr U, Pick L. Cofactor-interaction motifs and the cooption of a homeotic Hox protein into the segmentation pathway of Drosophila melanogaster Curr Biol CB. 2005;15:643-9.

91. Heffer A, Shultz JW, Pick L. Surprising flexibility in a conserved Hox transcription factor over 550 million years of evolution. Proc Natl Acad Sci U S A. 2010;107:18040-5.

92. Smith ST, Jaynes JB. A conserved region of engrailed, shared among all en-, gsc-, Nk1-, Nk2- and msh-class homeoproteins, mediates active transcriptional repression in vivo. Dev Camb Engl. 1996:122:3141-50.

93. Tolkunova EN, Fujioka M, Kobayashi M, Deka D, Jaynes JB. Two distinct types of repression domain in engrailed: one interacts with the groucho corepressor and is preferentially active on integrated target genes. Mol Cell Biol. 1998;18:2804-14.

94. Hittinger CT, Carroll SB. Evolution of an insect-specific GROUCHO-interaction motif in the ENGRAILED selector protein. Evol Dev. 2008;10:537-45.

95. Brayer KJ, Lynch VJ, Wagner GP. Evolution of a derived protein-protein interaction between HoxA11 and Foxola in mammals caused by changes in intramolecular regulation. Proc Natl Acad Sci U S A. 2011;108:E414-20.

96. Lynch VJ, Tanzer A, Wang Y, Leung FC, Gellersen B, Emera D, et al. Adaptive changes in the transcription factor HoxA-11 are essential for the evolution of pregnancy in mammals. Proc Natl Acad Sci U S A. 2008;105:14928-33.

97. Lynch VJ, Brayer K, Gellersen B, Wagner GP. HoxA-11 and FOXO1A cooperate to regulate decidual prolactin expression: towards inferring the core transcriptional regulators of decidual genes. PLoS One. 2009;4:e6845.

98. Van Loosdregt J, Coffer PJ. Post-translational modification networks regulating FOXP3 function. Trends Immunol. 2014:35:368-78.
99. Prasad MS, Sauka-Spengler T, LaBonne C. Induction of the neural crest state: Control of stem cell attributes by gene regulatory, post-transcriptional and epigenetic interactions. Dev Biol. 2012;366:10-21.

100. Kim DS, Hahn Y. Gains of ubiquitylation sites in highly conserved proteins in the human lineage. BMC Bioinformatics. 2012;13:306.

101. Kim DS, Hahn Y. Identification of novel phosphorylation modification sites in human proteins that originated after the human-chimpanzee divergence. Bioinforma Oxf Engl. 2011;27:2494-501.

102. Enard W, Przeworski M, Fisher SE, Lai CSL, Wiebe V, Kitano T, et al. Molecular evolution of FOXP2, a gene involved in speech and language. Nature. 2002:418:869-72.

103. Tsui D, Vessey JP, Tomita H, Kaplan DR, Miller FD. FoxP2 regulates neurogenesis during embryonic cortical development. J Neurosci Off J Soc Neurosci. 2013;33:244-58.

104. Ronshaugen M, McGinnis N, McGinnis W. Hox protein mutation and macroevolution of the insect body plan. Nature. 2002;415:914-7.

105. Taghli-Lamallem O, Hsia C, Ronshaugen M, McGinnis W. Context-dependent regulation of Hox protein functions by CK2 phosphorylation sites. Dev Genes Evol. 2008;218:321-32.

106. Bourbon HM, Martin-Blanco E, Rosen D, Kornberg TB. Phosphorylation of the Drosophila engrailed protein at a site outside its homeodomain enhances DNA binding. J Biol Chem. 1995;270:11130-9.

107. Jaffe L, Ryoo HD, Mann RS. A role for phosphorylation by casein kinase II in modulating Antennapedia activity in Drosophila. Genes Dev. 1997:11:1327-40.

108. Lynch VJ, May G, Wagner GP. Regulatory evolution through divergence of a phosphoswitch in the transcription factor CEBPB. Nature. 2011;480:383-6.

109. Maricic T, Günther V, Georgiev O, Gehre S, Curlin M, Schreiweis C, et al. A recent evolutionary change affects a regulatory element in the human FOXP2 gene. Mol Biol Evol. 2013;30:844-52.

110. Coolon JD, McManus CJ, Stevenson KR, Graveley BR, Wittkopp PJ. Tempo and mode of regulatory evolution in Drosophila. Genome Res. 2014;24:797-808.

doi:10.1186/2041-9139-6-3

Cite this article as: Cheatle Jarvela and Hinman: Evolution of transcription factor function as a mechanism for changing metazoan developmental gene regulatory networks. EvoDevo 2015 6:3.

\section{Submit your next manuscript to BioMed Central and take full advantage of:}

- Convenient online submission

- Thorough peer review

- No space constraints or color figure charges

- Immediate publication on acceptance

- Inclusion in PubMed, CAS, Scopus and Google Scholar

- Research which is freely available for redistribution 